\title{
Improving the output voltage waveform of an intense electron-beam accelerator based on helical type Blumlein pulse forming line
}

\author{
Xin-Bing Cheng, Jin-Liang Liu, Hong-Bo Zhang, Jia-Huai Feng, and Bao-Liang Qian \\ College of Opto-electronic Science and Engineering, National University of Defense Technology, Changsha, Hunan, 410073, \\ People's Republic of China \\ (Received 28 April 2010; published 27 July 2010)
}

\begin{abstract}
The Blumlein pulse forming line (BPFL) consisting of an inner coaxial pulse forming line (PFL) and an outer coaxial PFL is widely used in the field of pulsed power, especially for intense electron-beam accelerators (IEBA). The output voltage waveform determines the quality and characteristics of the output beam current of the IEBA. Comparing with the conventional BPFL, an IEBA based on a helical type BPFL can increase the duration of the output voltage in the same geometrical volume. However, for the helical type BPFL, the voltage waveform on a matched load may be distorted which influences the electron-beam quality. In this paper, an IEBA based on helical type BPFL is studied theoretically. Based on telegrapher equations of the BPFL, a formula for the output voltage of IEBA is obtained when the transition section is taken into account, where the transition section is between the middle cylinder of BPFL and the load. From the theoretical analysis, it is found that the wave impedance and transit time of the transition section influence considerably the main pulse voltage waveform at the load, a step is formed in front of the main pulse, and a sharp spike is also formed at the end of the main pulse. In order to get a well-shaped square waveform at the load and to improve the electron-beam quality of such an accelerator, the wave impedance of the transition section should be equal to that of the inner PFL of helical type BPFL and the transit time of the transition section should be designed as short as possible. Experiments performed on an IEBA with the helical type BPFL show reasonable agreement with theoretical analysis.
\end{abstract}

DOI: 10.1103/PhysRevSTAB.13.070402

PACS numbers: $84.30 . \mathrm{Ng}, 84.70 .+\mathrm{p}, 87.56 . \mathrm{bd}$

\section{INTRODUCTION}

The Blumlein pulse forming line (BPFL) [1] provides flat (rectangular) voltage pulses with an amplitude equal to the charging voltage when the load resistance is twice the impedance of the individual pulse forming lines (PFL). For the conventional cylindrical form BPFL, the pulse duration is the two-way transit time of the PFL [2]. To generate a pulse with duration of $200 \mathrm{~ns}$, the length of the PFL is about $20 \mathrm{~m}$ when transformer oil is used as the dielectric material. If instead, water with resistivity of more than $10 \mathrm{M} \Omega \mathrm{cm}$ is employed as the dielectric material of the PFL, its length is reduced to about $3.3 \mathrm{~m}$. This makes the BPFL very cumbersome and expensive. Also, for the water BPFL with length $3.3 \mathrm{~m}$, it is very difficult to keep the water resistivity high, with low resistivity, the radial current of the PFL would increase and the voltage would decrease. For high power [3,4], long pulse duration [5,6], and compact structure $[7,8]$, the helical type PFL is widely investigated. At the beginning of this century, the Institute of High Current Electronics combined a tesla transformer with a helical PFL, which made a compact and reliable high current beam accelerator [9]. The length of the PFL is only $4.2 \mathrm{~m}$ with transformer oil as dielectric material, but the duration of the output voltage is up to $130 \mathrm{~ns}$. Recently, an electron-beam accelerator based on the helical type BPFL with water dielectric was investigated [10], the length of the PFL was only $1.35 \mathrm{~m}$, but the duration of output pulse was about $200 \mathrm{~ns}$. The helical PFL is therefore a good way to increase the duration of the output voltage. The spiral PFL is a kind of slow wave structure. The spiral cylinder is a structure of axial symmetry and the gap spacing between the spiral strips is much shorter than the width of the spiral strip. The direction of the current on the spiral cylinder is along the spiral angle of the spiral strip, so the transit time of the PFL increases.

Intense electron-beam accelerators (IEBA) based on the conventional Blumlein PFL have been widely investigated by researchers [11-13], and the conventional cylindrical form BPFL consists of mainly three coaxial cylinders of different radii, named inner cylinder, middle cylinder, and outer cylinder, respectively $[14,15]$. The inner cylinder and middle cylinder form the inner PFL, the middle cylinder and outer cylinder form the outer PFL. For the helical type BPFL, the middle cylinder is usually a helical type cylinder [16]. Comparing with the conventional BPFL, a helical type BPFL can increase the duration of the output pulse in the same geometrical dimensions. Meanwhile, for the helical type BPFL, the transit time of inner PFL may be not equal to that of the outer PFL, causing the plateau region of the output voltage waveform of IEBA on the matched load to become distorted. In Ref. [16], the design of a helical BPFL for the ideal output voltage pulse on a matched load is discussed. A good output voltage waveform is obtained when the radius of the middle cylinder is the geometric average of the inner and outer cylinder radii. This design is 
for ideal conditions, but the helical type BPFL may be nonideal in many ways. The helical middle cylinder of BPFL results in unequal transit times for the inner and outer PFLs affecting the output voltage waveform. Other factors that can affect the output voltage were not considered in Ref. [16], such as the transition section. In Ref. [17], the effect of the transition section between the main switch and the middle cylinder of the BPFL is discussed. In Ref. [18], the effect of the transition section between the conventional BPFL and a matched load is reported; the transition section has a significant effect on the output voltage. The effect of transition section between the helical type BPFL and a load has not been reported when the transit time of the inner PFL is not equal to that of the outer PFL.

In this paper, a formula for calculating the main pulse of the helical type BPFL is obtained, and the effect of the transition section between the helical type BPFL and the matched load on the output voltage of IEBA is analyzed. The theoretical analysis is compared with experiments performed on and IEBA based on helical type BPFL with water dielectric. The experimental results are in agreement with the theoretical results, confirming that the output voltage waveform of an IEBA is improved significantly by optimizing the transition section, improving the flatness of the plateau region of the output waveform.

\section{THEORETICAL ANALYSIS}

\section{A. Model and theoretical calculation}

The construction of the IEBA based on helical type BPFL is illustrated in Fig. 1 which consists of the primary storage capacitor, field distortion switch [19], pulsed transformer, main switch, helical type BPFL, transition section, the load, and resistive divider. The only difference from a traditional IEBA is that a helical middle cylinder is used. The three coaxial cylindrical lines are filled with deionized water as dielectric. The transit time and wave impedance of the transition section are $\tau_{2}$ and $Z_{2}$.

Figure 2 shows the structure of the helical type BPFL and photograph of the helical middle cylinder. $r_{1}, r_{2}$, and $r_{3}$ are the radii of the inner, helical middle, and outer cylin-

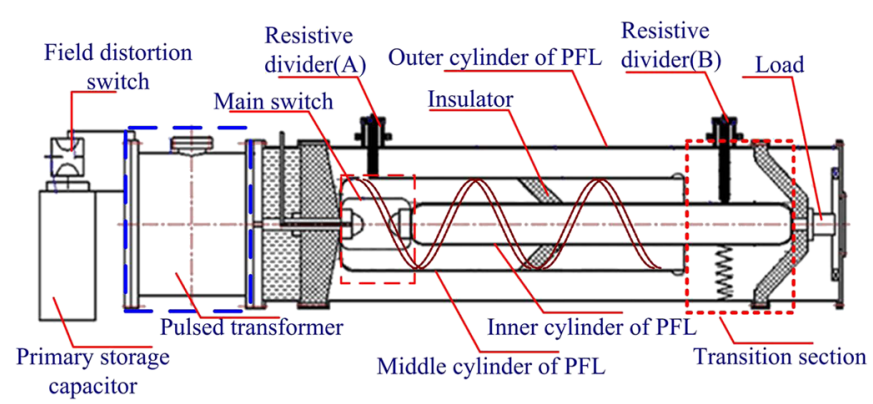

FIG. 1. (Color) Diagram of the IEBA based on helical type BPFL.

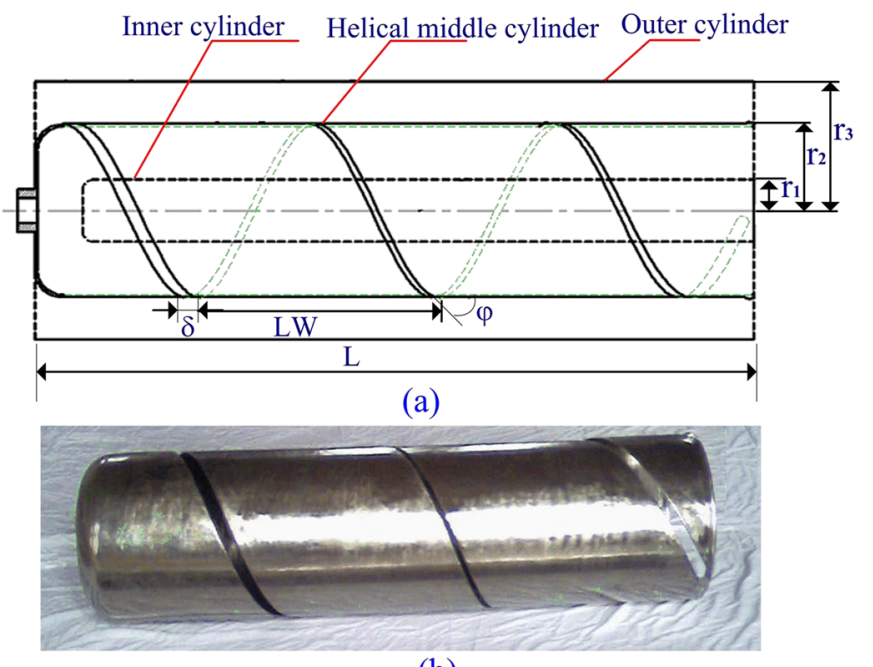

(b)

FIG. 2. (Color) Structure of the helical BPFL and photograph of the helical middle cylinder.

ders, respectively. LW is width of the spiral strip, $\mathrm{L}$ is the length of the PFL, $\delta$ is the $d$ turn-turn gap spacing of the spiral strips, $\varphi$ is the spiral angle. The PFL formed by the inner cylinder and the middle helical cylinders is called the inner PFL, and the outer PFL is formed by the middle helical cylinder and the outer cylinder. The transit time and wave impedance of the inner PFL and outer PFL are $\tau_{1}, Z_{1}$ and $\tau_{3}, Z_{3}$, respectively. These parameters can be calculated from Ref. [16] if the geometric parameters of the helical BPFL are known.

For the IEBA, during the helical BPFL discharge into the load, the equivalent circuit of the BPFL is shown in Fig. 3. S is the main switch, CE is the inner PFL, FD is the outer PFL, EA and BF is transition section, and $Z_{L}$ is the load. The BPFL is charged to a voltage $V_{B}(<0)$, when the main switch $\mathrm{S}$ is closed, the BPFL cannot stay in equilibrium, the capacitance of the inner line close to the switch starts discharging first. The voltage at the inner PFL and outer PFL satisfied the telegrapher equations:

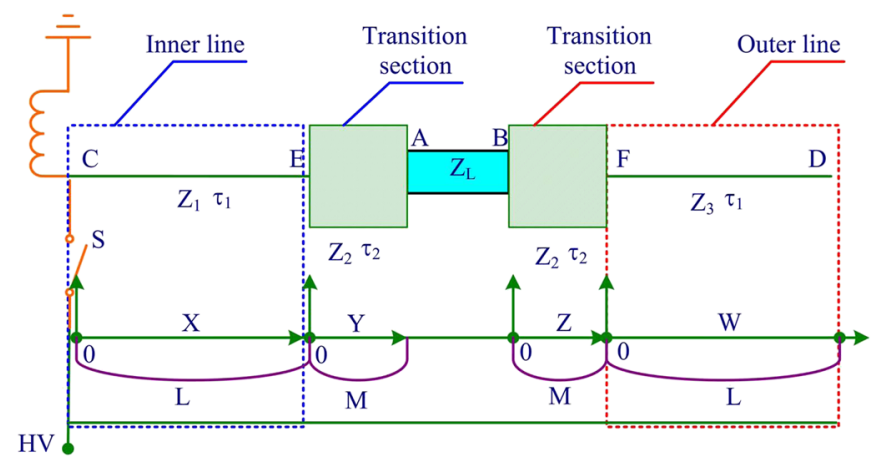

FIG. 3. (Color) Equivalent circuit of the helical type BPFL discharge into the load. 


$$
\begin{array}{cc}
-\frac{\partial u_{1}(X, t)}{\partial X}=L_{1} \frac{\partial i_{1}(X, t)}{\partial t} & -\frac{\partial u_{2}(Y, t)}{\partial Y}=L_{2} \frac{\partial i_{2}(Y, t)}{\partial t} \\
-\frac{\partial i_{1}(X, t)}{\partial X}=C_{1} \frac{\partial u_{1}(X, t)}{\partial t} & -\frac{\partial i_{2}(Y, t)}{\partial Y}=C_{2} \frac{\partial u_{2}(Y, t)}{\partial t} \\
-\frac{\partial u_{3}(Z, t)}{\partial Z}=L_{2} \frac{\partial i_{3}(Z, t)}{\partial t} & -\frac{\partial u_{4}(W, t)}{\partial W}=L_{3} \frac{\partial i_{4}(W, t)}{\partial t} \\
-\frac{\partial i_{3}(Z, t)}{\partial Z}=C_{2} \frac{\partial u_{3}(Z, t)}{\partial t} & -\frac{\partial i_{4}(W, t)}{\partial W}=C_{3} \frac{\partial u_{4}(W, t)}{\partial t},
\end{array}
$$

where $u_{1}(X, t), i_{1}(X, t)$ are the voltage and current of the inner PFL, $u_{2}(Y, t), i_{2}(Y, t), u_{3}(Z, t), i_{3}(Z, t)$, are the voltage and current of the transition sections, $u_{4}(W, t), i_{4}(W, t)$ are the voltage and current of the outer PFL. $L_{1}, L_{2}, L_{3}, C_{1}$, $C_{2}, C_{3}$ are the inductance and capacitance per unit of the inner PFL, transition section and outer PFL, respectively.
For the characteristic of the BPFL in Fig. 3, the initial and boundary conditions of the BPFL are

$$
\begin{aligned}
& \text { point } \mathrm{C} \rightarrow u_{1}(0, t)=0 \\
& \text { point } \mathrm{E} \rightarrow i_{1}(L, t)=i_{2}(0, t) \\
& \qquad \begin{aligned}
& u_{1}(L, t)=u_{2}(0, t) \\
& \text { point } \mathrm{A} \rightarrow i_{2}(M, t)=i_{3}(0, t) \\
& u_{2}(M, t)-u_{3}(0, t)=i_{3}(0, t) \times Z_{L}
\end{aligned} \\
& \text { point } \mathrm{F} \rightarrow i_{3}(M, t)=i_{4}(0, t) \\
& \qquad \begin{aligned}
u_{3}(M, t) & =u_{4}(0, t) \\
\text { point } \mathrm{D} \rightarrow i_{4}(L, t) & =0 .
\end{aligned}
\end{aligned}
$$

Then Laplace transformation is used to solve Eq. (1). The current and voltages are

$$
\begin{aligned}
& i_{1}(X, s)=A_{1} e^{-\left(s \tau_{1} / L\right) X}+B_{1} e^{\left(s \tau_{1} / L\right) X} \rightarrow u_{1}(X, s)=\frac{V_{B}}{s}+Z_{1}\left(A_{1} e^{-\left(s \tau_{1} / L\right) X}-B_{1} e^{\left(s \tau_{1} / L\right) X}\right) \\
& i_{2}(Y, s)=A_{2} e^{-\left(s \tau_{2} / M\right) Y}+B_{2} e^{\left(s \tau_{2} / M\right) Y} \rightarrow u_{2}(Y, s)=\frac{V_{B}}{s}+Z_{2}\left(A_{2} e^{-\left(s \tau_{2} / M\right) Y}-B_{2} e^{\left(s \tau_{2} / M\right) Y}\right) \\
& i_{3}(Z, s)=A_{3} e^{-\left(s \tau_{2} / M\right) Z}+B_{3} e^{\left(s \tau_{2} / M\right) Z} \rightarrow u_{3}(Z, s)=\frac{V_{B}}{s}+Z_{2}\left(A_{3} e^{-\left(s \tau_{2} / M\right) Z}-B_{3} e^{\left(s \tau_{2} / M\right) Z}\right) \\
& i_{4}(W, s)=A_{4} e^{-\left(s \tau_{3} / L\right) W}+B_{4} e^{\left(s \tau_{3} / L\right) W} \rightarrow u_{4}(W, s)=\frac{V_{B}}{s}+Z_{3}\left(A_{4} e^{-\left(s \tau_{3} / L\right) W}-B_{4} e^{\left(s \tau_{3} / L\right) W}\right),
\end{aligned}
$$

where $A_{1}, A_{2}, A_{3}, A_{4}, B_{1}, B_{2}, B_{3}, B_{4}$ are undetermined coefficients, and $s$ is the parameter in the Laplace transformation. $\tau_{1}=L \sqrt{L_{1} C_{1}}, \tau_{2}=M \sqrt{L_{2} C_{2}}, \tau_{3}=L \sqrt{L_{3} C_{3}}$, and $\tau_{1} \neq \tau_{3}$. Then substituting Eq. (3) into Eq. (2), and supposing that $x=e^{s \tau_{1}}, y=e^{s \tau_{2}}, z=e^{s \tau_{3}}$, the output voltage of IEBA is

$$
\begin{aligned}
u_{Z_{L}}(s)= & \frac{2 V_{B} Z_{L}}{s} \frac{\Theta+\Gamma}{\Lambda} \\
\Theta= & \left(\beta_{5}-\beta_{1}\right) x^{-1} y^{-1}+\left(\beta_{6}-\beta_{3}\right) x^{-1} y^{-3} \\
\Gamma= & \left(\beta_{7}-\beta_{2}\right) x^{-1} y^{-1} z^{-2}+\left(\beta_{8}-\beta_{4}\right) x^{-1} y^{-3} z^{-2} \\
\Lambda= & \alpha_{1} x^{-2} y^{-4} z^{-2}+\alpha_{2} y^{-4} z^{-2}+\alpha_{3} x^{-2} z^{-2}+\alpha_{4} z^{-2} \\
& +\alpha_{5} x^{-2} y^{-4}+\alpha_{6} y^{-4}+\alpha_{7} x^{-2}+\alpha_{8} \\
& +\alpha_{9} x^{-2} y^{-2} z^{-2}-\alpha_{10} y^{-2} z^{-2}+\alpha_{10} x^{-2} y^{-2} \\
& -\alpha_{9} y^{-2}
\end{aligned}
$$

and there are

$$
\begin{array}{ll}
\beta_{1}=\left(Z_{L}+2 Z_{2}\right)\left(Z_{2}+Z_{3}\right) & \beta_{2}=-\left(Z_{L}+2 Z_{2}\right)\left(Z_{2}-Z_{3}\right) \\
\beta_{3}=Z_{L}\left(Z_{2}-Z_{3}\right) & \beta_{4}=-Z_{L}\left(Z_{2}+Z_{3}\right) \\
\beta_{5}=Z_{L}\left(Z_{2}+Z_{3}\right) & \beta_{6}=\left(Z_{L}-2 Z_{2}\right)\left(Z_{2}-Z_{3}\right) \\
\beta_{7}=-Z_{L}\left(Z_{2}-Z_{3}\right) & \beta_{8}=-\left(Z_{L}-2 Z_{2}\right)\left(Z_{2}+Z_{3}\right)
\end{array}
$$

$$
\begin{array}{ll}
\alpha_{1}=-\left(Z_{L}-2 Z_{2}\right)\left(Z_{1}+Z_{2}\right)\left(Z_{2}+Z_{3}\right) & \alpha_{2}=\left(Z_{L}-2 Z_{2}\right)\left(Z_{1}-Z_{2}\right)\left(Z_{2}+Z_{3}\right) \\
\alpha_{3}=\left(Z_{L}+2 Z_{2}\right)\left(Z_{1}-Z_{2}\right)\left(Z_{2}-Z_{3}\right) & \alpha_{4}=-\left(Z_{L}+2 Z_{2}\right)\left(Z_{1}+Z_{2}\right)\left(Z_{2}-Z_{3}\right) \\
\alpha_{5}=\left(Z_{L}-2 Z_{2}\right)\left(Z_{1}+Z_{2}\right)\left(Z_{2}-Z_{3}\right) & \alpha_{6}=-\left(Z_{L}-2 Z_{2}\right)\left(Z_{1}-Z_{2}\right)\left(Z_{2}-Z_{3}\right) \\
\alpha_{7}=-\left(Z_{L}+2 Z_{2}\right)\left(Z_{1}-Z_{2}\right)\left(Z_{2}+Z_{3}\right) & \alpha_{8}=\left(Z_{L}+2 Z_{2}\right)\left(Z_{1}+Z_{2}\right)\left(Z_{2}+Z_{3}\right) \\
\alpha_{9}=-2 Z_{L}\left(Z_{2}^{2}-Z_{1} Z_{3}\right) & \alpha_{10}=2 Z_{L}\left(Z_{2}^{2}+Z_{1} Z_{3}\right) .
\end{array}
$$


For the IEBA, the main pulse is the most important and we pay more attention to the flatness of the main pulse of the output voltage of IEBA. So during the inverse Laplace transformation of Eq. (4), just the $x^{-1} y^{-n}, x^{-3} y^{-n}$, and $x^{-1} y^{-n} z^{-2}$ are taken into account. Then Eq. (4) can be further simplified as

$$
\begin{aligned}
u_{Z_{L}}(s)= & u_{1}+u_{2}-u_{3}+O\left(\tau_{1}, \tau_{2}, \tau_{3}\right) \\
u_{1}= & \frac{2 V_{B} Z_{L} \Theta}{s \alpha_{8}} \sum_{n=0}^{\infty} \sum_{i=0}^{n} \sum_{j=0}^{i} \sum_{k=0}^{n-i} \sum_{l=0}^{n-i-k}(-1)^{n} C_{n}^{i} C_{i}^{j} C_{n-i-k}^{l}\left(\frac{\alpha_{6}}{\alpha_{8}}\right)^{j}\left(-\frac{\alpha_{9}}{\alpha_{8}}\right)^{i-j}\left(\frac{\alpha_{7}}{\alpha_{8}}\right)^{k}\left(\frac{\alpha_{5}}{\alpha_{8}}\right)^{l}\left(\frac{\alpha_{10}}{\alpha_{8}}\right)^{n-i-k-l} x^{-2(n-i)} y^{-2(n+j+l-k)} \\
u_{2}= & -\frac{2 V_{B} Z_{L}(\Theta+\Gamma)}{s \alpha_{8}} \sum_{n=0}^{\infty} \sum_{i_{1}=0}^{n} \sum_{j_{1}=0}^{i_{1}} \sum_{k_{1}=0}^{n-i_{1}} \sum_{l_{1}=0}^{n-i_{1}-k_{1}}(-1)^{n} C_{n}^{i_{1}} C_{i_{1}}^{j_{1}} C_{n-i_{1}-k_{1}}^{l_{1}}\left(\frac{\alpha_{6}}{\alpha_{8}}\right)^{j_{1}}\left(-\frac{\alpha_{9}}{\alpha_{8}}\right)^{i_{1}-j_{1}}\left(\frac{\alpha_{4}}{\alpha_{8}}\right)^{k_{1}}\left(\frac{\alpha_{2}}{\alpha_{8}}\right)^{l_{1}}\left(-\frac{\alpha_{10}}{\alpha_{8}}\right)^{n-i_{1}-k_{1}-l_{1}} \\
& \times z^{-2\left(n-i_{1}\right)} y^{-2\left(n+j_{1}+l_{1}-k_{1}\right)} \\
u_{3}= & \frac{2 V_{B} Z_{L} \Theta}{s \alpha_{8}} \sum_{n=0}^{\infty} \sum_{i_{2}=0}^{n}(-1)^{n} C_{n}^{i_{2}}\left(\frac{\alpha_{6}}{\alpha_{8}}\right)^{i_{2}}\left(-\frac{\alpha_{9}}{\alpha_{8}}\right)^{n-i_{2}} y^{-2\left(n-i_{2}\right)} .
\end{aligned}
$$

Equation (7) determines the main pulse of the output voltage, where $O\left(\tau_{1}, \tau_{2}, \tau_{3}\right)$ stands for the remainder. If $t<$ $\max \left[3 \tau_{1}+3 \tau_{2}, \tau_{1}+3 \tau_{2}+2 \tau_{3}\right]$, then $O\left(\tau_{1}, \tau_{2}, \tau_{3}\right)=0$. $C_{n}^{i}$ is a symbol of binomial. $C_{n}^{i}=\frac{n !}{i ! \times(n-i) !}$. Using the characteristic of the inverse Laplace transformation,

$$
L^{-1}\left(\frac{e^{-s n \tau}}{s}\right)=\lambda(t-n \tau)
$$

where $L^{-1}$ stands for inverse Laplace transformation, $\lambda(t)$ is the step function, defined as

$$
\lambda(t)= \begin{cases}1 & t \geq 0 \\ 0 & t<0\end{cases}
$$

In fact, according to the characteristic of wave propagation, if just the main pulse is considered, the calculation time $\left(T_{C}\right)$ is finite. The remainder operator $O\left(\tau_{1}, \tau_{2}, \tau_{3}\right)$ is zero if the time is bigger than $T_{C}$. Of course, since the calculation time is finite, $n$ is finite for the main pulse of the output voltage of the IEBA, and $n$ is determined by $\tau_{1}, \tau_{2}$, and $\tau_{3}$. For the main pulse, it is easy to find that $n_{\max }=$ $\max \left[\tau_{1} / \tau_{2}, \tau_{3} / \tau_{2}\right]$. So the main pulse of the output voltage of the IEBA is

$$
\begin{aligned}
u_{Z_{L}}(\mathrm{t})= & u_{1}+u_{2}-u_{3}+O\left(\tau_{1}, \tau_{2}, \tau_{3}\right) \\
u_{1}= & \frac{2 V_{B} Z_{L}}{\alpha_{8}} \sum_{n=0}^{n_{\max }} \sum_{i=0}^{n} \sum_{j=0}^{i} \sum_{k=0}^{n-i} \sum_{l=0}^{n-i-k}(-1)^{n} C_{n}^{i} C_{i}^{j} C_{n-i-k}^{l}\left(\frac{\alpha_{6}}{\alpha_{8}}\right)^{j}\left(-\frac{\alpha_{9}}{\alpha_{8}}\right)^{i-j}\left(\frac{\alpha_{7}}{\alpha_{8}}\right)^{k}\left(\frac{\alpha_{5}}{\alpha_{8}}\right)^{l}\left(\frac{\alpha_{10}}{\alpha_{8}}\right)^{n-i-k-l} H_{1} \\
H_{1}= & \left(\beta_{5}-\beta_{1}\right) \lambda\left(t-2(n-i+0.5) \tau_{1}-2(n+j+l-k+0.5) \tau_{2}\right)+\left(\beta_{6}-\beta_{3}\right) \lambda\left(t-2(n-i+0.5) \tau_{1}\right. \\
& \left.-2(n+j+l-k+1.5) \tau_{2}\right) \\
u_{2}= & -\frac{2 V_{B} Z_{L}}{\alpha_{8}} \sum_{n=0}^{n_{\max }} \sum_{i_{1}=0}^{n} \sum_{j_{1}=0}^{i_{1}} \sum_{k_{1}=0}^{n-i_{1}} \sum_{l_{1}=0}^{n-i_{1}-k_{1}}(-1)^{n} C_{n}^{i_{1}} C_{i_{1}}^{j_{1}} C_{n-i_{1}-k_{1}}^{l_{1}}\left(\frac{\alpha_{6}}{\alpha_{8}}\right)^{j_{1}}\left(-\frac{\alpha_{9}}{\alpha_{8}}\right)^{i_{1}-j_{1}}\left(\frac{\alpha_{4}}{\alpha_{8}}\right)^{k_{1}}\left(\frac{\alpha_{2}}{\alpha_{8}}\right)^{l_{1}}\left(-\frac{\alpha_{10}}{\alpha_{8}}\right)^{n-i_{1}-k_{1}-l_{1}} H_{2} \\
H_{2}= & \left(\beta_{5}-\beta_{1}\right) \lambda\left(t-\tau_{1}-2\left(n+j_{1}+l_{1}-k_{1}+0.5\right) \tau_{2}-2\left(n-i_{1}\right) \tau_{3}\right) \\
& +\left(\beta_{6}-\beta_{3}\right) \lambda\left(t-\tau_{1}-2\left(n+j_{1}+l_{1}-k_{1}+1.5\right) \tau_{2}-2\left(n-i_{1}\right) \tau_{3}\right) \\
& +\left(\beta_{7}-\beta_{2}\right) \lambda\left(t-\tau_{1}-2\left(n+j_{1}+l_{1}-k_{1}+0.5\right) \tau_{2}-2\left(n-i_{1}+1\right) \tau_{3}\right) \\
& +\left(\beta_{8}-\beta_{4}\right) \lambda\left(t-\tau_{1}-2\left(n+j_{1}+l_{1}-k_{1}+1.5\right) \tau_{2}-2\left(n-i_{1}+1\right) \tau_{3}\right) \\
u_{3}= & \frac{2 V_{B} Z_{L}}{\alpha_{8}} \sum_{n=0}^{n_{\max }} \sum_{i_{2}=0}^{n}(-1)^{n} C_{n}^{i_{2}}\left(\frac{\alpha_{6}}{\alpha_{8}}\right)^{i_{2}}\left(-\frac{\alpha_{9}}{\alpha_{8}}\right)^{n-i_{2}} H_{3} \\
H_{3}= & \left(\beta_{5}-\beta_{1}\right) \lambda\left(t-\tau_{1}-2\left(n-i_{2}+0.5\right) \tau_{2}\right)+\left(\beta_{6}-\beta_{3}\right) \lambda\left(t-\tau_{1}-2\left(n-i_{2}+1.5\right) \tau_{2}\right) .
\end{aligned}
$$


Substituting the parameters of the helical type BPFL into Eq. (10), the main pulse of the output voltage of the IEBA can be obtained.

\section{B. Discussion with different conditions}

According to the analysis above and Eq. (10), it is obvious that the output voltage of the IEBA is dependent on the wave impedance and the transit time of the helical type BPFL and the transition section between the BPFL and the matched load. Here, the effect of the transition section on the output voltage is discussed by changing parameters of the transition section.

\section{Wave impedance of the transition section}

First, suppose that the wave impedance of the inner PFL is equal to that of outer PFL, and the load is matched with the BPFL, which means that $Z_{1}=Z_{3}, Z_{L}=Z_{1}+Z_{3}$. In order to show the output voltage waveform for a specific case, it is assumed that $Z_{1}=Z_{3}=6 \Omega, Z_{L}=12 \Omega, \tau_{1}=$ $80 \mathrm{~ns}, \tau_{2}=12 \mathrm{~ns}$, and $\tau_{3}=90 \mathrm{~ns}$. Substituting these parameters into Eq. (10), we can obtain the output voltage of the IEBA for different transition section impedances.
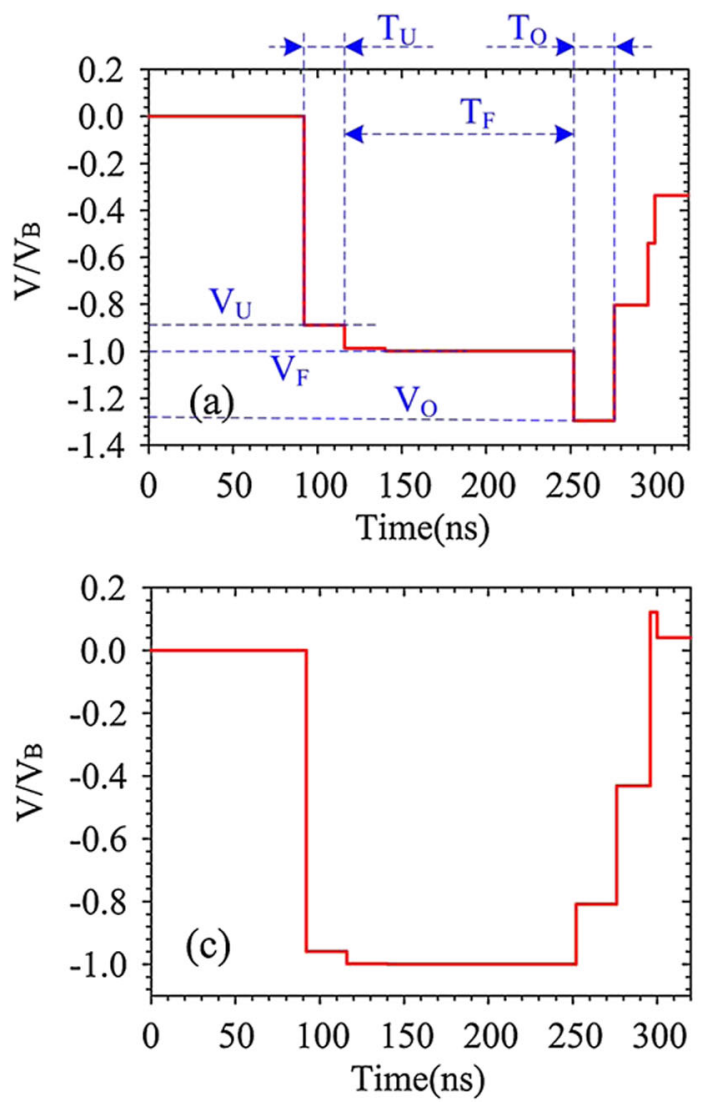

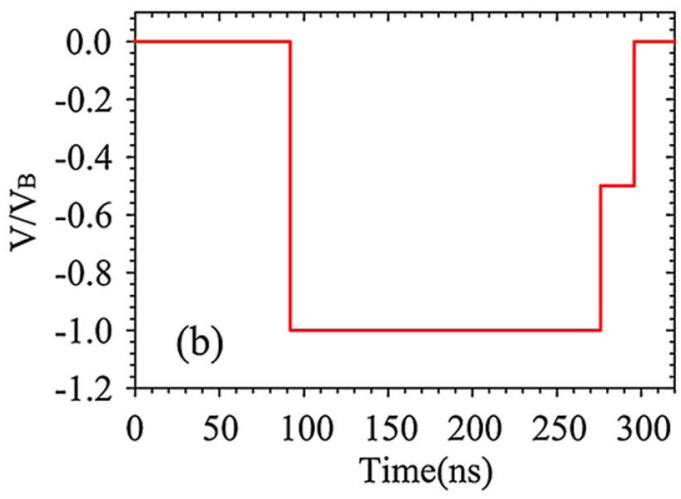

Figure 4 shows the main pulse of the output voltage when the impedance of the transition section $\left(Z_{2}\right)$ is $3,6,9$, and $12 \Omega$, respectively. It clearly shows that there are steps (undershoot) in front of the main pulse. Just as in Fig. 4(a), the voltage of undershoot is $V_{U}$ with duration of $T_{U}$. But when the impedance of the transition section is equal to that of the inner PFL of the helical type BPFL, the step is almost nonexistent and the output voltage waveform is the flattest, as shown in Fig. 4(b). Meanwhile, if the wave impedance of the transition section is too small, there will be an overshoot at the end of the main pulse, it is shown in Fig. 4(a), and the voltage of the overshoot is $V_{O}$ with duration of $T_{O}$. Also, if the wave impedance of the transition section is too big, the fall time of the output voltage will be increased and the plateau time $\left(T_{F}\right)$ of the main pulse is decreased. For example, if $Z_{2}$ is $6 \Omega$, the fall time of the main pulse is about $20 \mathrm{~ns}$ with plateau time of $184 \mathrm{~ns}$, but when $Z_{2}$ is $12 \Omega$, the fall time is up to $44 \mathrm{~ns}$ with plateau time of $160 \mathrm{~ns}$. So for the helical type BPFL, a proper wave impedance of the transition section is very important for forming a well-shaped square wave on a matched load. For a square wave, the flatness the flatness $\left(\eta_{F}\right)$ could be defined as the ration of the deviant and the

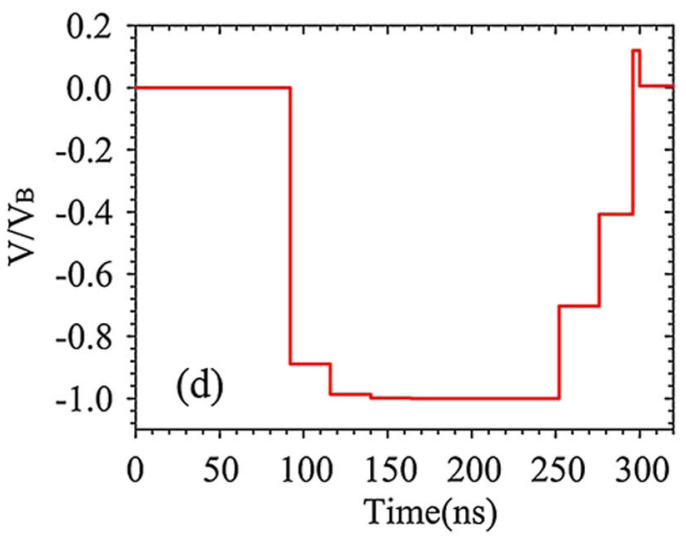

FIG. 4. (Color) The main pulse of the output voltage at different wave impedance of the transition section, in all cases, $\tau_{1}=80 \mathrm{~ns}$, $\tau_{2}=12 \mathrm{~ns}, \tau_{3}=90 \mathrm{~ns}, Z_{1}=Z_{3}=6 \Omega, Z_{L}=12 \Omega$, the wave impedance of the transition section is varied: (a) $Z_{2}=3 \Omega$, (b) $Z_{2}=6 \Omega$, (c) $Z_{2}=9 \Omega$, and (d) $Z_{2}=12 \Omega$. 
flat part $\left(V_{F}\right)$ of the output voltage, so

$$
\eta_{F 1}=\frac{V_{U}-V_{F}}{V_{F}}, \quad \eta_{F 2}=\frac{V_{O}-V_{F}}{V_{F}}
$$

In Eq. (11), $\eta_{F 1}$ is negative and $\eta_{F 2}$ is positive. For a well-shaped output voltage, there should be no undershoot and no overshoot, so $\eta_{F 1}=\eta_{F 2}=0$ as shown in Fig. 4(b). Also, the duration is also an important parameter, in Fig. 4(a), if the duration of the square wave is a constant, the smaller $T_{U}$ and $T_{O}$, the better the square wave.

In fact, though the IEBA is designed as $Z_{1}=Z_{3}$, the wave impedance of inner and outer PFL may be not equal, because $Z_{1}$ and $Z_{3}$ may be changed by many factors, such as the distortion of the helical middle cylinder of BPFL and the error of machining. So, it is very important to discuss the condition that $Z_{1} \neq Z_{3}$, but we also assume that the load is matched with the BPFL. Now, it is supposed that $Z_{1}=7 \Omega, Z_{3}=5 \Omega, Z_{L}=12 \Omega$ and $\tau_{1}=80 \mathrm{~ns}, \tau_{2}=$ $12 \mathrm{~ns}, \tau_{3}=90 \mathrm{~ns}$. The output voltage of the IEBA for different transition section impedances are obtained from Eq. (10). Figure 5 shows the calculation results when the impedance of the transition section is $3,5,7$, and $12 \Omega$, respectively.

In Fig. 5, the output voltage waves are similar to those in Fig. 4, steps also exist in front of the main pulse, but for a smaller $Z_{2}$, there are more steps in front of the main pulse. In Fig. 5(a), three steps exist, so $T_{U}$ is increased. Also, an overshoot exists when the wave impedance of the transition section is small, and the amplitude of the overshoot increases as $Z_{2}$ decreases, as shown in Figs. 5(a) and 5(b). Meanwhile, when the wave impedance of the transition section is big enough, the overshoot is almost nonexistent, as shown in Figs. 5(c) and 5(d). Comparing Fig. 5(c) with Fig. 5(d), the fall time increases as $Z_{2}$ further increases, and the plateau time of the main pulse decreases. So the output voltage of Fig. 5(c) is much better, and $\eta_{F 1}$ is $-7.7 \%, \eta_{F 2}$ is about zero. These examples prove that a proper wave impedance of the transition section is very important for forming a well-shaped square wave, and the wave impedance of the transition section should be equal to that of the inner PFL of the helical type BPFL.

\section{Transit time of the transition section}

In Eq. (10), it is clearly shown that the main pulse of the output voltage is also determined by the transit time of the transition section, so in order to analyze the effect of the transition section on the output voltage, it is very important to discuss the effect of the transit time of the transition section on the output voltage. Supposing that $Z_{1}=7 \Omega$, $Z_{2}=6 \Omega, \quad Z_{3}=5 \Omega, \quad Z_{L}=12 \Omega, \quad \tau_{1}=80 \mathrm{~ns}$,
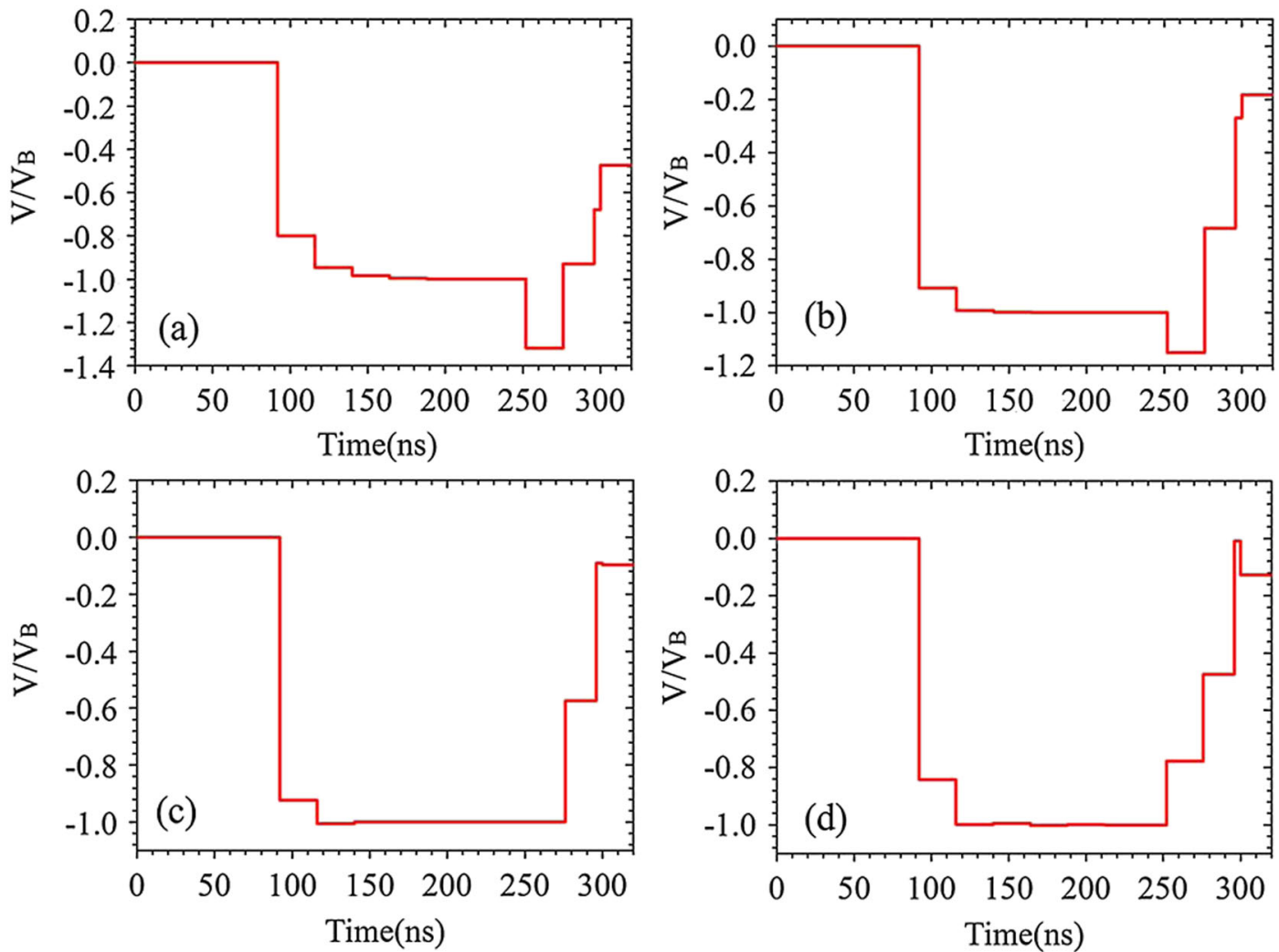

FIG. 5. (Color) The main pulse of the output voltage at different wave impedance of the transition section, in all cases, $\tau_{1}=80 \mathrm{~ns}$, $\tau_{2}=12 \mathrm{~ns}, \tau_{3}=90 \mathrm{~ns}, Z_{1}=7 \Omega, Z_{3}=5 \Omega, Z_{L}=12 \Omega$, the wave impedance of the transition section is varied: (a) $Z_{2}=3 \Omega$, (b) $Z_{2}=5 \Omega$, (c) $Z_{2}=7 \Omega$, and (d) $Z_{2}=12 \Omega$. 


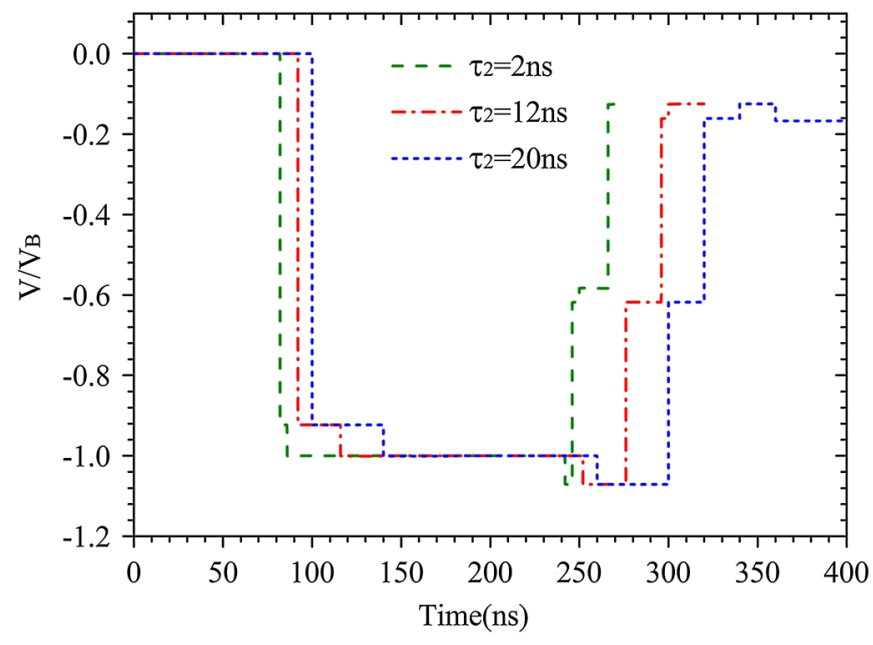

FIG. 6. (Color) The main pulse of the output voltage at different transit time of the transition section when $\tau_{1}=80 \mathrm{~ns}, \tau_{3}=$ $90 \mathrm{~ns}, Z_{1}=7 \Omega, Z_{2}=6 \Omega, Z_{3}=5 \Omega, Z_{L}=12 \Omega$.

$\tau_{3}=90 \mathrm{~ns}$, and the transit time of the transition section is 2,12 , and $20 \mathrm{~ns}$, respectively. Then submitting these parameters into Eq. (10), the main pulse of the output voltage is obtained in Fig. 6.

In Fig. 6, the flatness $\eta_{F 1}$ are all $-0.77 \%$ and $\eta_{F 2}$ are all $7.1 \%$, but $T_{U}, T_{F}$, and $T_{O}$ are all different. For example, if the transit time of the transition section is $2 \mathrm{~ns}$, the output voltage is a well-shaped square wave, the duration of the step $\left(T_{U}\right)$ which is in front of the main pulse is about $4 \mathrm{~ns}$, $T_{F}$ is about $156 \mathrm{~ns}$, and $T_{O}$ is about $4 \mathrm{~ns}$. However, as the increment of the transit time of the transition section, it is up to $20 \mathrm{~ns}$, the duration of the step $\left(T_{U}\right)$ is increased to $40 \mathrm{~ns}$. At the same time, the duration of the overshoot at the end of the main pulse is also increased to $40 \mathrm{~ns}$, and $T_{F}$ is only $120 \mathrm{~ns}$. Though the full width at half maximum (FWHM) of the output voltage is increased, the output voltage waveform is distorted and the plateau time is decreased. So for the same helical type BPFL, the transit time of the transition section should be short, the shorter the better. Otherwise, the output voltage waveform will be affected. Also, we know that the transit time of the transition section is $\tau_{2}=M \frac{\sqrt{\varepsilon_{r}}}{c}\left(\varepsilon_{r}\right.$ is the relative permittivity of the dielectric of the BPFL, $c$ is the velocity of light), so the length of the transition section should be kept as short as possible.

Through the above analysis, some basic conclusion can be obtained. (1) A proper wave impedance of the transition section is very important for forming a well-shaped square wave, and the wave impedance of the transition section should be equal to that of the inner PFL of helical type BPFL. (2) For the same helical type BPFL, the transit time of the transition section should be short, so the length of the transition section should be kept as short as possible, which also helps to generate a well-shaped square wave with minimal distortion.

\section{EXPERIMENTAL RESULT AND DISCUSSION}

In order to identify the correctness of the theoretical analysis above, an experiment is performed using an IEBA based on the helical type BPFL with water dielectric in our laboratory, the structure of the IEBA is shown in Fig. 1. The operating process of the IEBA is as follows. When the charging voltage of the primary storage capacitor of $12 \mu \mathrm{F}$ reached to a certain value, the field distortion switch was triggered and closed, and then the primary storage capacitor discharged to the primary winding of the transformer. Consequently, the transformer started to charge the BPFL. Once the charging voltage of the BPFL reaches the breakdown value of the main switch, the PFL discharged to the load. In the experiments, the radius of the outer, helical middle, and inner cylinders are 275, 200, and $110 \mathrm{~mm}$, repetitively. The length of the PFL is $1280 \mathrm{~mm}$; the spiral angle is $60^{\circ}$. The number of spiral turn is 2.2 . Through the theoretical calculation from Ref. [16], the wave impedance of the inner PFL and outer PFL are about 6.4 and $4 \Omega$, respectively. The transit time $\tau_{1}$ is about $70 \mathrm{~ns}$ and $\tau_{3}$ is $76 \mathrm{~ns}$. The wave impedance and transit time of the transition section are $6.1 \Omega$ and $15 \mathrm{~ns}$, respectively. During the experiments, a high power water load with inductance of $20 \mathrm{nH}$ and capacitance of $0.5 \mathrm{nF}$ is used, and the resistance of the load is adjustable. The output voltage of the IEBA is measured by a resistance voltage divider and the current is measured by a Rogowski coil. Figure 7 shows the output voltage waveform when the load is $12 \Omega$.

Figure 7 shows that the output peak voltage and current of the IEBA are about $380 \mathrm{kV}$ and $36 \mathrm{kA}$, with FWHM of about $200 \mathrm{~ns}$. The output voltage waveform has a step in front of the main pulse, and the voltage and duration of the step is about $340 \mathrm{kV}$ and $30 \mathrm{~ns}$, respectively. So the flatness $\eta_{F 1}$ is about $-10.5 \%$. Substituting the parameters of the

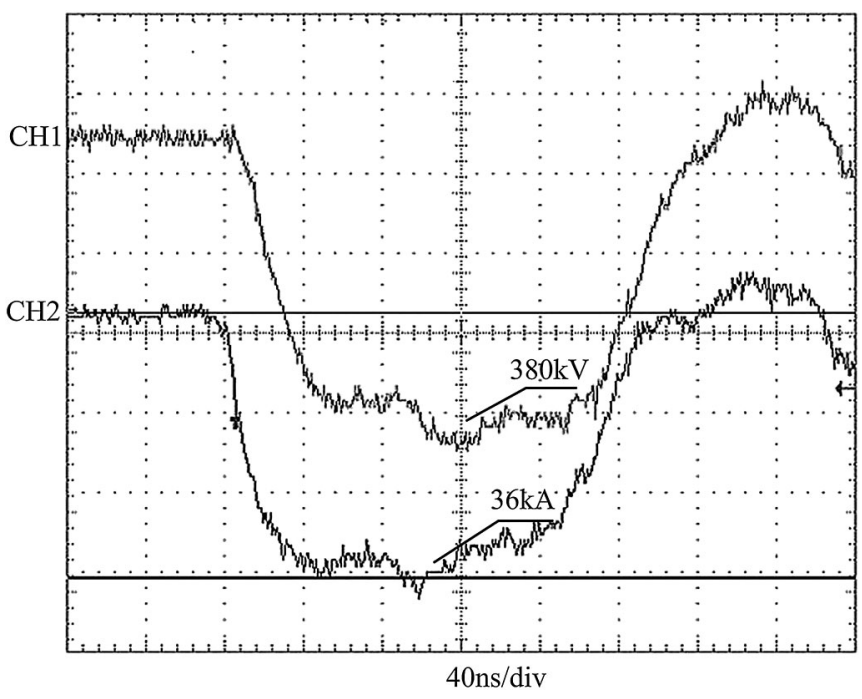

FIG. 7. Output voltage $(\mathrm{CH} 1)$ and current $(\mathrm{CH} 2)$ of the IEBA in the experiment, $\tau_{1}=70 \mathrm{~ns}, \tau_{2}=15 \mathrm{~ns}, \tau_{3}=76 \mathrm{~ns}, Z_{1}=$ $6.4 \Omega, Z_{2}=6.1 \Omega, Z_{3}=4 \Omega, Z_{L}=12 \Omega$. 


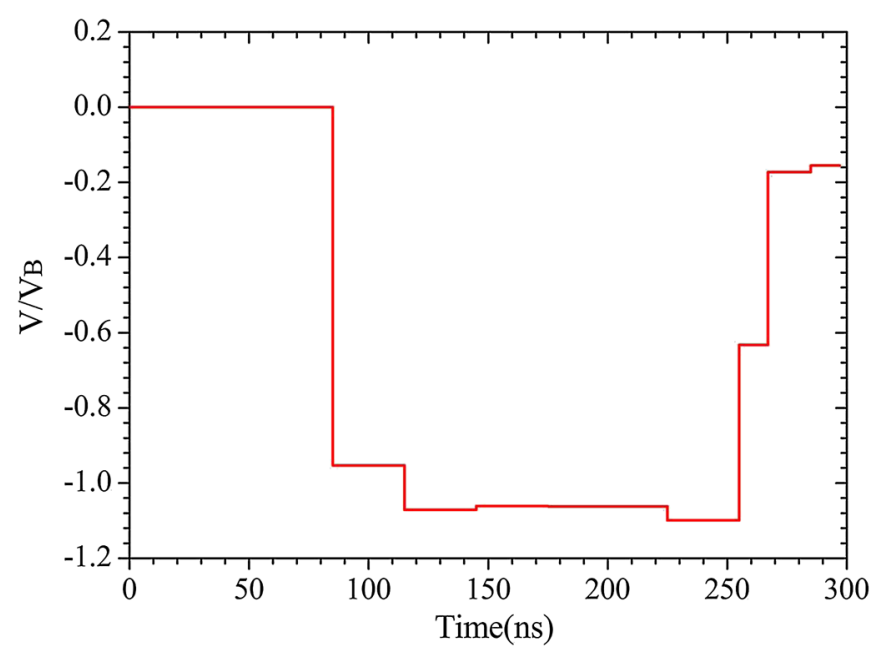

FIG. 8. (Color) Output voltage of the IEBA from the theoretical calculation when $\tau_{1}=70 \mathrm{~ns}, \tau_{2}=15 \mathrm{~ns}, \tau_{3}=76 \mathrm{~ns}, Z_{1}=$ $6.4 \Omega, Z_{2}=6.1 \Omega, Z_{3}=4 \Omega, Z_{L}=12 \Omega$.

IEBA into the Eq. (10), the theoretical output voltage of the IEBA is shown in Fig. 8.

Comparing with Fig. 7, the theoretical output voltage compares well with the experimental result. For example, there is a step in front of the main pulse, and the duration of the step is about $30 \mathrm{~ns}$. Also, there is a much smaller step which is adjacent to the $30 \mathrm{~ns}$ step. Second, the FWHM of the main pulse is about $200 \mathrm{~ns}$. It is noted that there are also small differences between the experiment result and theoretical calculation, due to the stray inductance of the load, the main switch and other stray parameter are ignored in the theoretical calculation. But the transition section is the main reason why the step occurs prior to the main pulse; it can be proved as follows.

In order to further verify the conclusion above, the same experiment was performed using the IEBA except the length of the transition section was reduced, the same high power water load is used. The transit time of the transition section $\tau_{2}$ was about $5 \mathrm{~ns}$. The experimental output voltage of the IEBA is shown in Fig. 9.

The output voltage waveform in Fig. 9 is very similar to that in Fig. 7. The output voltage also has its own characteristics. For example, there is also a step in front of the main pulse, but the duration of the step is obviously decreased, from $40 \mathrm{~ns}$ to about $10 \mathrm{~ns}$. Also, in Fig. 9, the output voltage is a well-shaped square waveform and there is little fluctuation on the flat part of the output voltage, which is beneficial for IEBA applications, such as high power microwave generators (HPMG). The typical HPMG can operate at the highest efficiency with a flattop rectangular pulse [20]. In Fig. 9, there is a very small sharp spike at the end of the main pulse, which is similar to that in Fig. 7; this is because that the wave impedance of the transition section is approximately equal to that of the inner PFL. These phenomena are also very clearly observed from the current waveform in Figs. 7 and 9.

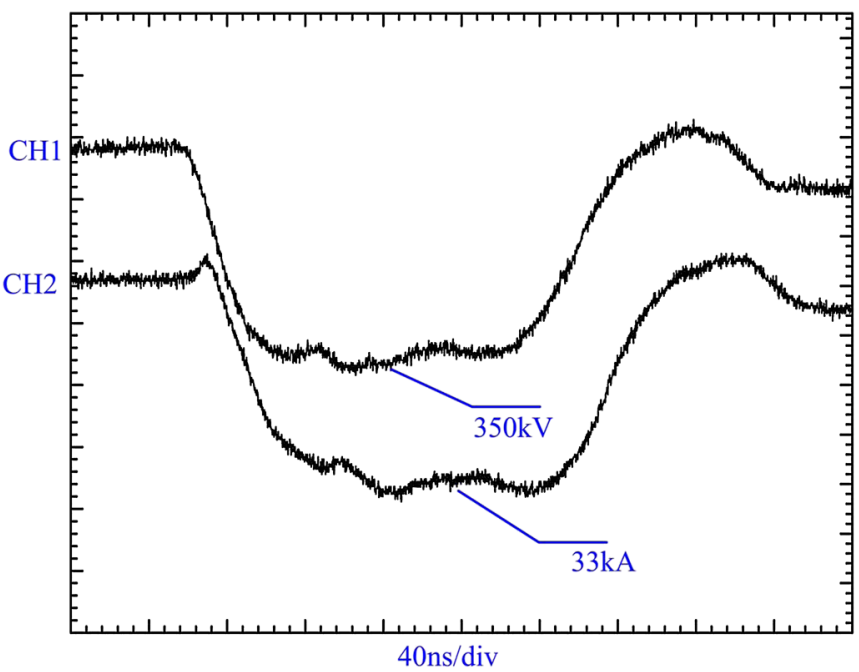

FIG. 9. (Color) Output voltage $(\mathrm{CH} 1)$ and current $(\mathrm{CH} 2)$ of the IEBA in the experiment after the transition section is amended, $\tau_{1}=70 \mathrm{~ns}, \tau_{2}=5 \mathrm{~ns}, \tau_{3}=76 \mathrm{~ns}, Z_{1}=6.4 \Omega, Z_{2}=6.1 \Omega$, $Z_{3}=4 \Omega, Z_{L}=12 \Omega$.

Substituting these parameters into Eq. (10), the output voltage waveform is shown in Fig. 10. Comparing with Fig. 9, the theoretical output voltage agrees well with the experimental result. For the theoretical calculation results in Fig. 10, the duration and amplitude of the step all agree with the experimental results in Fig. 9, including the duration and amplitude of the sharp spike.

These results in Figs. 7 and 9 show that the transition section between the helical type BPFL and the load has a significant effect on the output voltage of the IEBA, and the experimental results correspond well with the theoretical analysis. Also, the output voltage waveform of the IEBA is improved by reducing the length of the transition section.

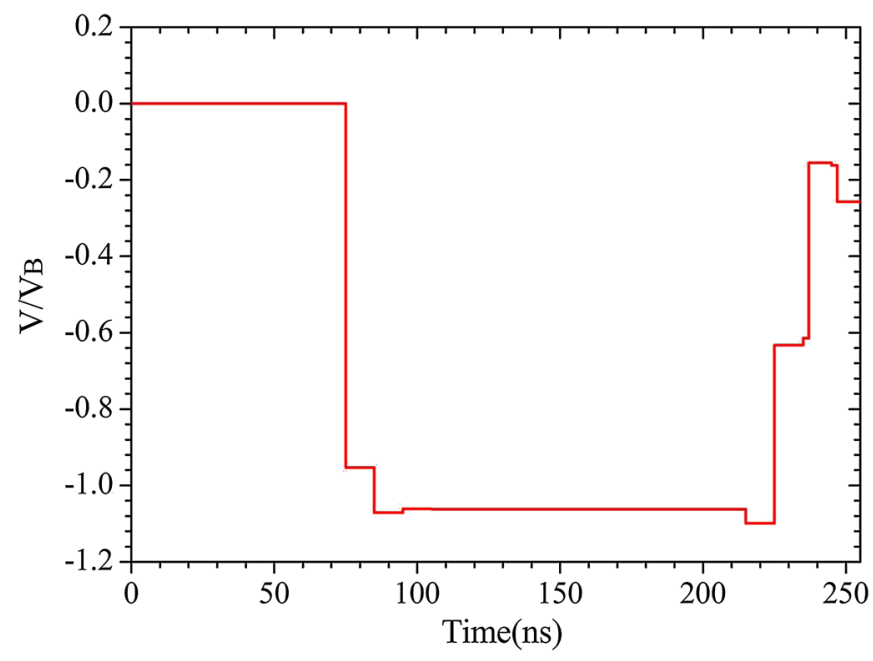

FIG. 10. (Color) Output voltage of the IEBA from the theoretical calculation after the transition section is amended, $\tau_{1}=$ $70 \mathrm{~ns}, \tau_{2}=15 \mathrm{~ns}, \tau_{3}=76 \mathrm{~ns}, Z_{1}=6.4 \Omega, Z_{2}=6.1 \Omega, Z_{3}=$ $4 \Omega, Z_{L}=12 \Omega$. 


\section{CONCLUSION}

The equivalent circuit of the helical type BPFL discharging into the load is modeled where the transition section between the BPFL and the load is taken into account. A formula for the output voltage of the IEBA is obtained, and the characteristics of the output voltage waveform are discussed for different conditions. Experiments are performed to compare with the theoretical results. Through the theoretical analysis and experimental results, it is found that the output voltage of IEBA based on helical type BPFL has the following characteristics: (1) The voltage waveform is affected by the transition section between the helical type BPFL and the load, and a step will be formed in front of the main pulse. At the same time, a sharp spike is also formed at the end of the main pulse. (2) The amplitude of the step and sharp spike are all related to the wave impedance of the transition section. In order to make the step and spike disappear, the wave impedance of the transition section should be equal to that of the inner PFL of helical type BPFL. In order to decrease the duration of the step and spike, the length of the transition section should be kept as short as possible. These all help to generate a wellshaped square wave.

\section{ACKNOWLEDGMENTS}

The authors gratefully acknowledge the support from the Fund of Innovation, Graduate school of NUDT under Grant No. B090701. This work is also supported by Hunan Provincial Innovation Foundation for Postgraduate. The authors would like to thank Zhi-Qiang Hong for his assistance in the experiment.

[1] A. D. Blumlein, U.S. Patent No. 2,496,979 (1950).

[2] S. T. Pai and Q. Zhang, Introduction To High Power pulse Technology (World Scientific, Singapore, 1995).

[3] V. P. Gubanov, S. D. Korovin, I. V. Pegel, A. M. Roitman, V. V. Rostov, and A. S. Stepchenko, IEEE Trans. Plasma Sci. 25, 258 (1997).

[4] D. H. H. Hoffmann, A. Blazevic, P. Ni, O. Rosmej, M. Roth, N. A. Tahir, A. Tauschwitz, S. Udrea, D. Varentsov, K. Weyrich, and Y. Maron, Laser Part. Beams 23, 47 (2005).
[5] D.S. Kumar and M. J. Thomas, in Proceedings of the 9th International Conference on Electromagnetic Interference and Compatibility, 2006 (Karnataka, India, 2006), pp. $433-437$.

[6] J. C. Su, X. B. Zhang, G. Z. Lui, X. X. Song, Y. F. Pan, L. M. Wang, J.C. Peng, and Z.J. Ding, IEEE Trans. Plasma Sci. 37, 1954 (2009).

[7] G. A. Mesyats, S. D. Korovin, V. V. Rostov, V. G. Shpak, and M. I. Yalandin, Proc. IEEE 92, 1166 (2004).

[8] Y. Zhang, J. L. Liu, X. B. Cheng, G. Q. Bai, H. B. Zhang, J.H. Feng, and B. Liang, Rev. Sci. Instrum. 81, 033302 (2010).

[9] S. D. Koroein, V. P. Gubanov, A. V. Gunin, I. V. Pegel, and A.S. Stepchenko, The 28th IEEE International Conference on Plasma Science, Las Vegas, 2001, pp. 1249-1251.

[10] J. L. Liu, Y. Yin, B. Ge, T. W. Zhan, X. B. Cheng, J. H. Feng, T. Shu, J.D. Zhang, and X.X. Wang, Laser Part. Beams 25, 593 (2007).

[11] S. Friedman, R. Limpaecher, and M. Sirchis, IEEE Conference Record of the Eighteenth Power Modulator Symposium (IEEE, Hilton Head, SC, 1988), pp. 360-366.

[12] S. Katsuki, D. Takano, T. Namihira, and H. Akiyama, Rev. Sci. Instrum. 72, 2759 (2001).

[13] J.L. Liu, C. L. Lu, J.D. Zhang, S. Li, and X. X. Wang, Laser Part. Beams 24, 355 (2006).

[14] T. Sakugawa, D. Wang, K. Shinozaki, T. Namihira, S. Katsuki, and H. Akiyama, in Proceedings of the 14th International Pulsed Power Conference (IEEE, New York, 2003), Vol. 1, pp. 657-660.

[15] Y. L. Cheng, Y. P. Zhao, B. Luan, L. Y. Han, Q. S. Zhu, and Q. Wang, in the 8th International Conference on Laser and Fiber-Optical Networks Modeling, 2006 (Kharkiv, Ukraine, 2006), pp. 62-65.

[16] J. L. Liu, X. B. Cheng, B. L. Qian, B. Ge, J. D. Zhang, and X. X. Wang, Laser Part. Beams 27, 95 (2009).

[17] X. B. Cheng, J. L. Liu, B. L. Qian, and J. D. Zhang, Laser Part. Beams 27, 439 (2009).

[18] X. B. Cheng, J. L. Liu, Y. Zhang, J. H. Feng, and B. L. Qian, Phys. Rev. ST Accel. Beams 12, 110401 (2009).

[19] X. B. Cheng, J. L. Liu, B. L. Qian, Z. Chen, and J. H. Feng, IEEE Trans. Plasma Sci. 38, 516 (2010).

[20] R. J. Barker and E. Schamiloglu, High Power Microwave Sources and Technologies, Institute of Electrical and Electronics Engineers, Inc. (Tsinghua University Press, Beijing, 2005) (in Chinese). 